\title{
Infraocclusion Treated with Removable Prosthesis on Occlusal Surface of Severely Attritioned Teeth
}

\author{
Akinori Tasaka, Takayuki Ueda, Kaoru Sakurai
}

\begin{abstract}
A 66-year-old man visited our department with the chief complaint of poor esthetics due to loss of a fixed partial denture in a maxillary incisor tooth and difficulty in mastication due to an ill-fitting, mandibular removable partial denture. An analysis of the occlusal vertical dimension resulted in a diagnosis of infraocclusion. After increasing vertical dimension with a provisional restoration and treatment denture which covered the attritioned surface, a definitive prosthesis was applied. Untreated loss of molars and severe attrition of the remaining teeth caused infraocclusion in this patient, which increased occlusal loading on the incisor teeth and induced detachment of the prosthesis in the maxillary incisors. Esthetic appearance was improved and masticatory dysfunction restored by increasing vertical dimension and oral rehabilitation. Mildly invasive prosthetic treatment involved application of a removable partial denture which covered the attritioned surface, not only infraocclusion but masticatory dysfunction and poor esthetics were also treated.
\end{abstract}

Keywords: Infraocclusion, Attritioned teeth, Vertical dimension increase, Removable partial denture, Occlusal reconstruction.

How to cite this article: Tasaka A, Ueda T, Sakurai K. Infraocclusion Treated with Removable P rosthesis on Occlusal Surface of Severely Attritioned Teeth. Int J Prosthodont Restor Dent 2012;2(2):66-71.

\section{Source of support $\mathrm{Nil}$}

Conflict of interest: None

\section{INTRODUCTION}

Failure to treat molar loss and attrition of the remaining teeth can make subsequent prosthetic treatment difficult. Extrusion of dental antagonists due to untreated tooth loss leads to vertical narrowing of the denture space, and shortening of the abutment teeth due to attrition induces a decrease in the vertical dimension of occlusion. Here, we report a patient with severe attrition of the remaining teeth in whom a satisfactory outcome was achieved by increasing the vertical dimension with a removable partial denture which covered the attritioned surface, compensating for infraocclusion.

\section{CASE REPORT}

The patient was a 66-year-old man who first visited our department on $M$ arch 23, 2009, with the chief complaint of poor esthetic appearance due to loss of a fixed partial denture of a maxillary incisor and difficulty in mastication due to an ill-fitting, removable partial denture in the mandible.

History of current denture: In 2007, the patient received a fixed partial denture at another clinic for loss of the maxillary left central incisor (\#21, FDI Two-Digit N otation). This denture used the maxillary right central and left lateral incisors as abutment teeth (\#11 and 22). In 2008, the fixed partial denture became detached and had to be recemented. Thereafter, the patient consulted the Department of Prosthetic Dentistry, Tokyo D ental College Chiba Hospital, as detachment of the fixed partial denture recurred. In 2006, the patient had al so received a mandibular bilateral extension base denture, which had been repeatedly adjusted or repaired at another clinic.

Intraoral findings: Teeth \#21, 36, 37, 46 and 47 were lost, and the occlusal support was B 2 according to Eichner's classification (Fig. 1). The remaining teeth in the maxillary dentition showed marked attrition, and the lingual cusp of the maxillary left premolar, in particular, was attritioned to the gingival margin. The lingual surfaces of the maxillary incisors also showed marked attrition (Fig. 2); deep bite was observed in the incisors, and clearance was markedly deficient. A ttrition was grade IV according to the Carlsson scale. ${ }^{1}$ A ccording to the classification system for partial edentulism of the Japan Prosthodontic Society, ${ }^{2}$ the condition was level II. The fixed partial denture was detached and the crow $n$ removed at \#22 and both the crown and metal core removed at \#11. Tooth \#11 also showed root fracture (Fig. 3). In the mandibular dentition, unilateral bounded saddle dentures were applied for bilateral molar Ioss. A n intermediary defect was observed on the right side, and the denture sank during occlusion due to breakage of the rest. The retainer of the left unilateral bounded saddle denture was broken, and the artificial teeth showed marked abrasion (Fig. 4). Irremediable caries was noted in \#45, to which a coping was applied. The periodontal pocket was $5 \mathrm{~mm}$ deep at \#11 but $3 \mathrm{~mm}$ or less at other sites, and the mobility of the remaining teeth was grade 0 . The opening and closing movement path showed no deviation, and no symptoms of temporomandibular disorder were noted.

\section{Diagnosis}

The direct cause of detachment of the fixed partial denture was determined to be marked attrition of the remaining teeth and increased occlusal loading of the incisors due to a lack of occlusal support in the molar region. The results of facial measurement (Willis's method) were as follows: The distance between the pupil and angulus oris was $72 \mathrm{~mm}$; 


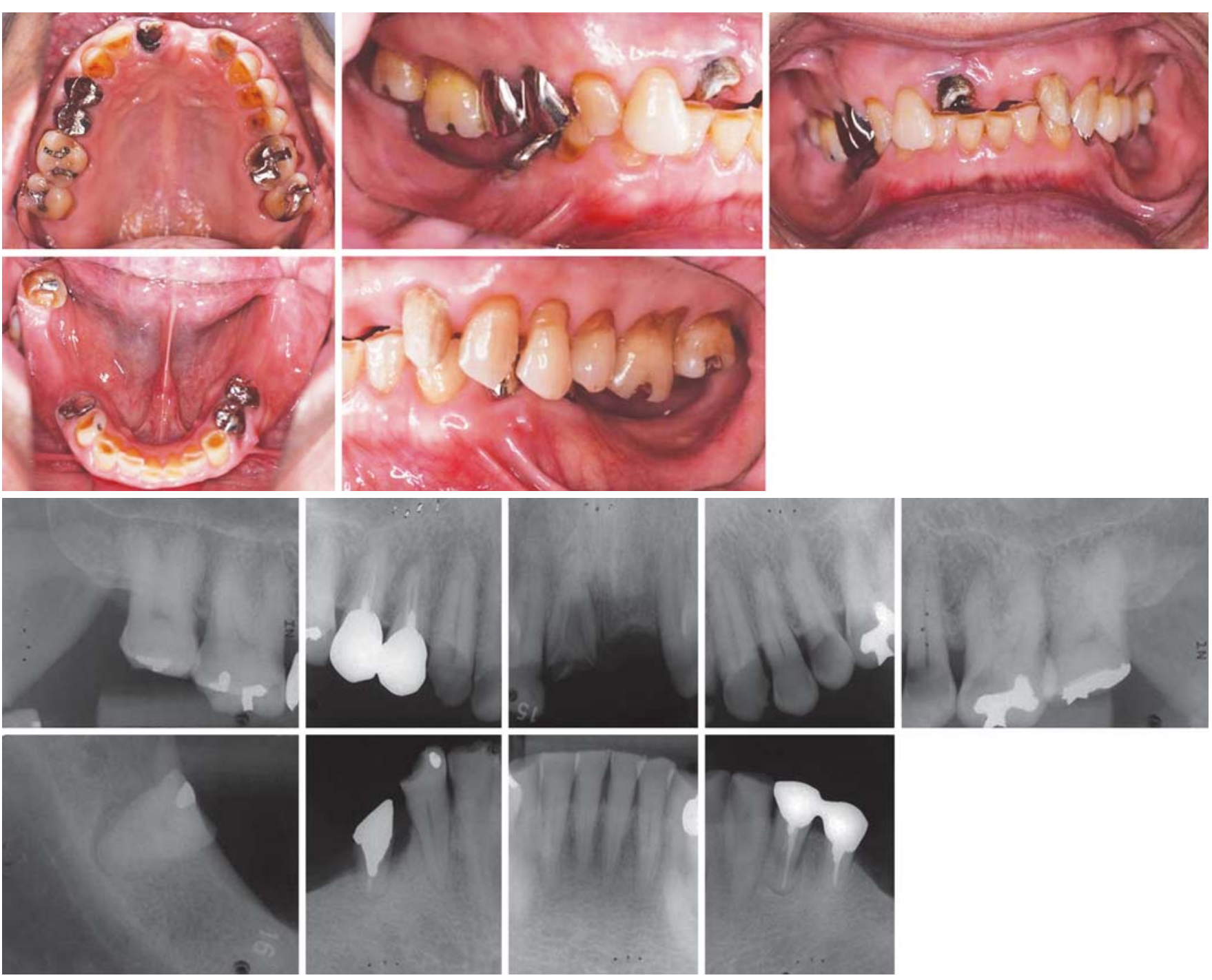

Fig. 1: Intraoral photographs and dental X-ray images obtained at initial examination

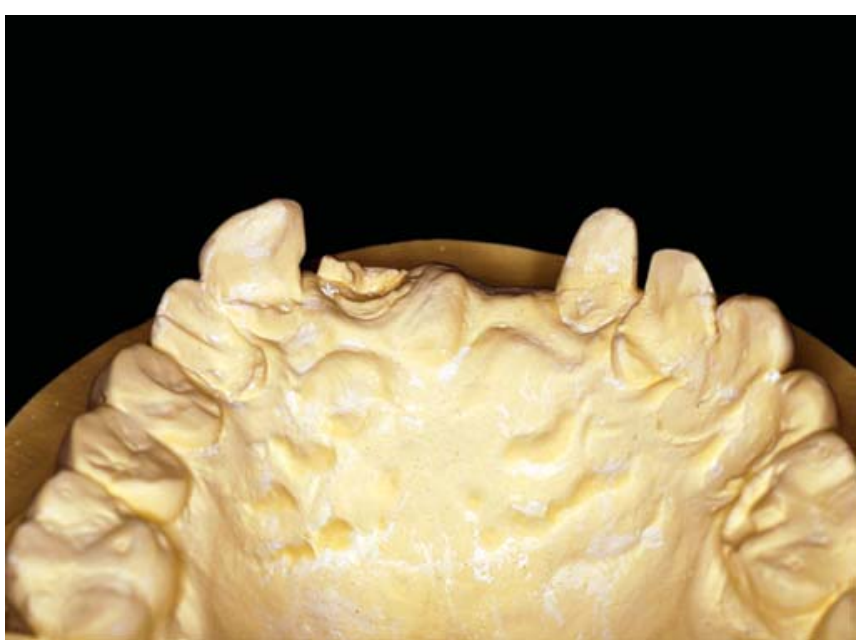

Fig. 2: Degree of attrition in maxillary dentition

and the distance between the subnasal point and submental region was $70 \mathrm{~mm}$ at rest position; during occlusion, the distance between the subnasal point and the submental region was $63 \mathrm{~mm}$. Cephalographic findings (Ricketts analysis) ${ }^{3}$ were as follows: Facial axis, 89; facial depth,

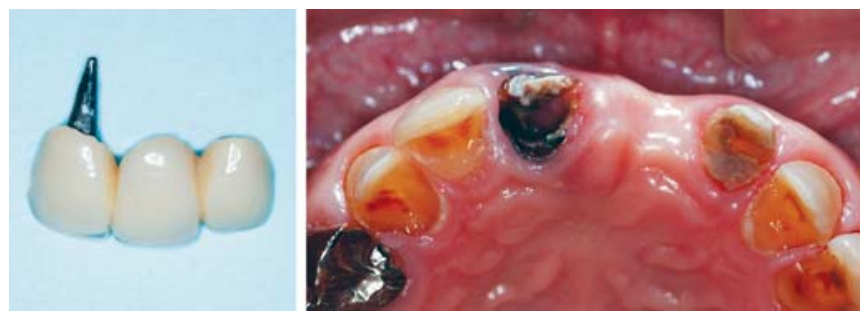

Fig. 3: Detached fixed partial denture and fractured upper right central incisor (at initial examination)

$86^{\circ}$; mandibular plane, $25^{\circ}$; lower facial height, $50^{\circ}$; and mandibular arc, $17^{\circ}$. A diagnosis of infraocclusion was made based on these findings. In terms of mastication disorder, the chewing score was $65 \%$, and chewing function was judged to be level IV according to the Sato evaluation. ${ }^{4}$ Teeth \#11 and \#45 were judged to be unsalvageable due to root fracture and irremediable caries, respectively.

\section{Therapeutic Strategy}

We decided to treat the infraocclusion by securing the denture space and clearance in the upper incisor region by 


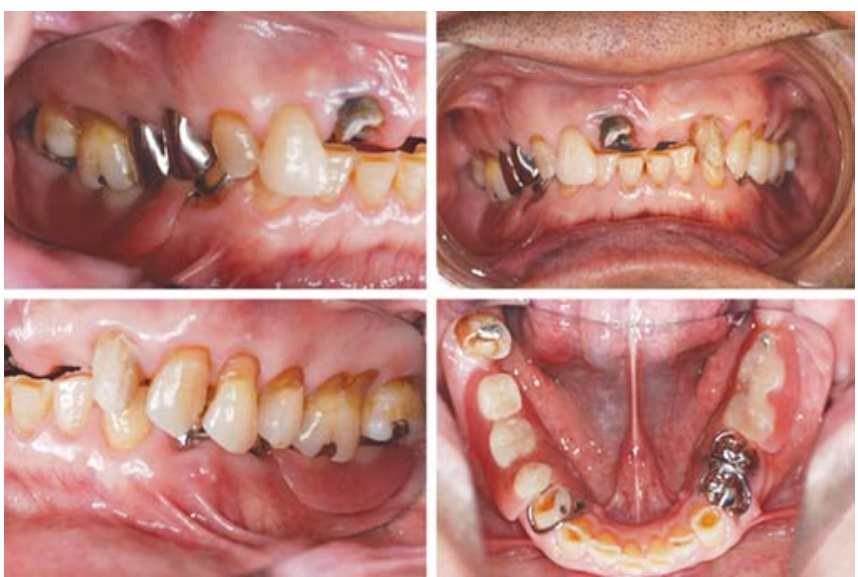

Fig. 4: Intraoral photographs of with dentures (at initial examination)

simultaneously increasing the vertical dimension and restoring occlusal support in the molar region. However, the patient was reluctant to undergo the suggested treatment after it was explained that it would be necessary to cut and carry out pulpectomy of multiple teeth if crown restoration was selected for restoration of the attritioned area of the maxillary dentition. A less invasive prosthetic treatment plan was then proposed which involved using a removable partial denture to cover the attritioned area and prosthetic treatment of teeth \#11 and 21. This time, the patient gave their consent. Thus, it was decided to first increase vertical dimension using a provisional restoration and a treatment denture. A fter confirming the esthetic outcome and state of the periodontal tissues and occlusion, we would then go on to perform definitive prosthetic treatment.

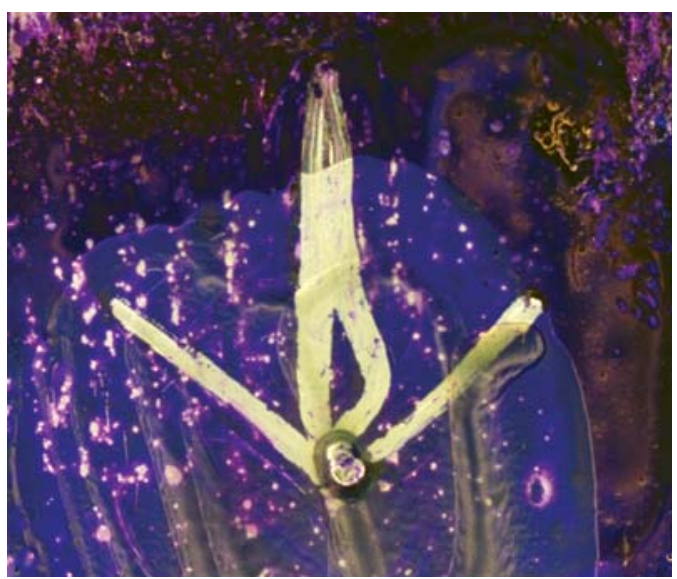

Fig. 5: Gothic arch trace and tapping point at vertical dimension increase

\section{Treatment and Follow-up}

Teeth \#11 and 45, which were judged to be unsalvageable, were extracted. The occlusal vertical dimension was then increased by $5 \mathrm{~mm}$ between the subnasal point and submental region by evaluating the results of facial measurements and the physiologic resting position of the mandible. A Gothic arch tracer was used to determine the horizontal mandibular position after the vertical dimension had been increased. The tapping point was set at the apex of the Gothic arch. Centric occlusion was set at the apex (Fig. 5). A diagnostic wax-up was performed and crown morphology, state of occlusal contact and prosthetic space confirmed (Fig. 6).

The esthetic appearance of the incisor was restored. A provisional restoration was applied to \#14,15 and 22 . The
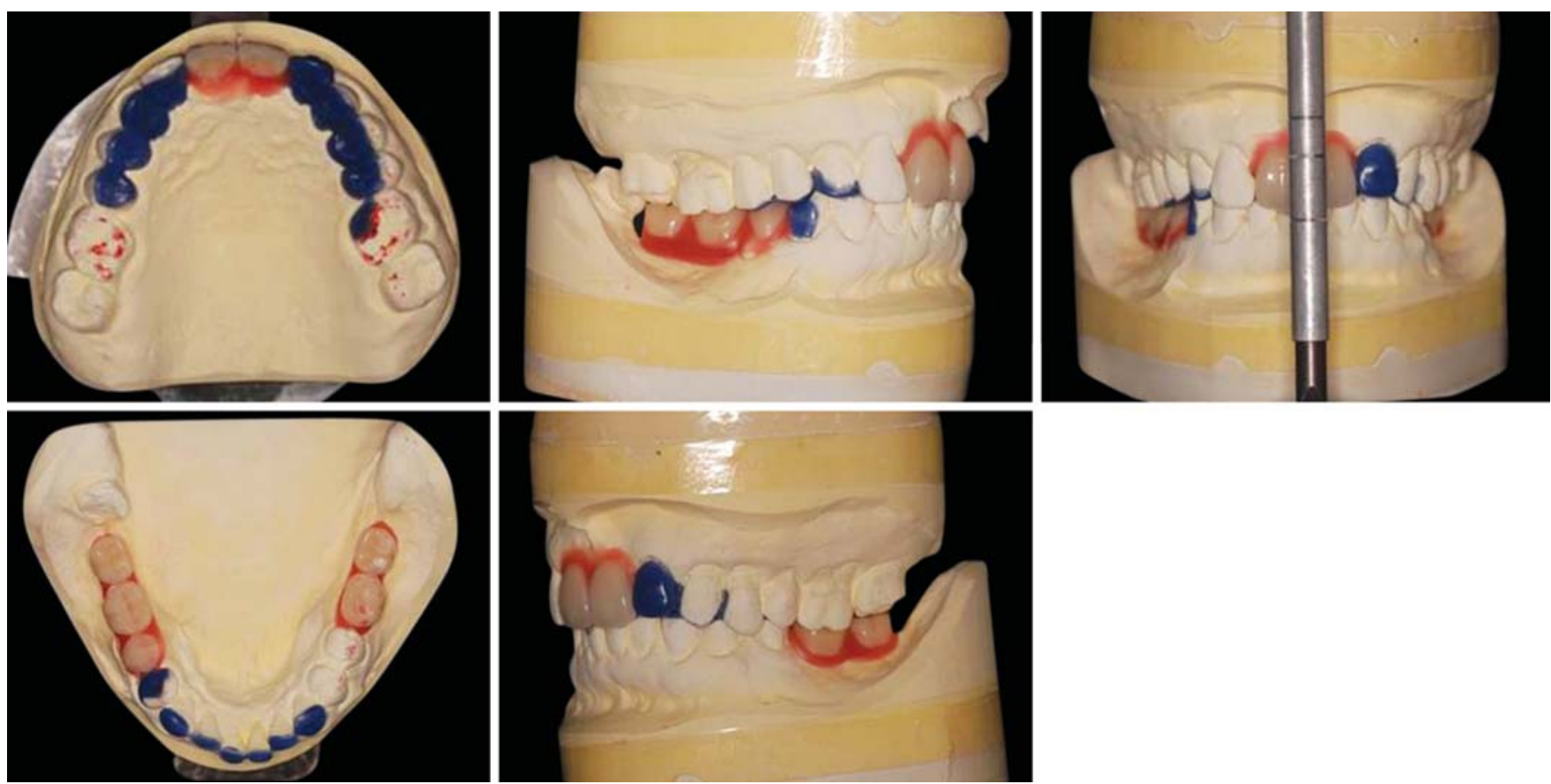

Fig. 6: Diagnostic wax-up after vertical dimension increase 

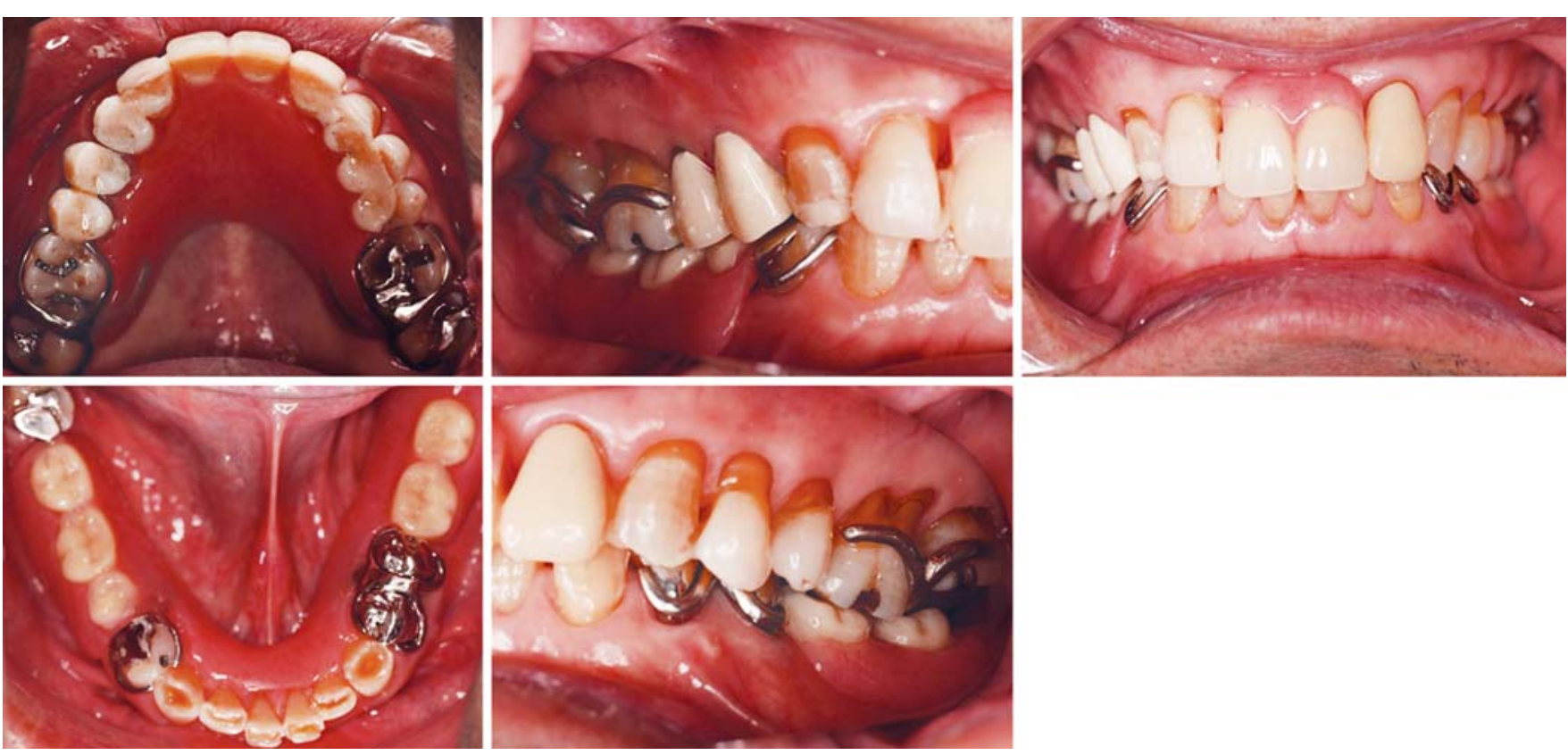

Fig. 7: Intraoral photographs obtained at after application of provisional restoration and treatment denture

surface of a treatment denture was covered with resin in those areas which would come into contact with the attritioned surfaces. A diagnostic wax-up was used as a reference. A bilateral removable partial denture was prepared for the defects at \#36, 37, 45, 46 and 47, and an onlay rest was set at the attritioned area of \#44 to simultaneously restore occlusal contact and prevent sinking of the denture base (Fig. 7). A ttrition of mandibular tooth remained untreated to request from the patient. G roup function was applied to the occlusal scheme. The patient reported frequent cheek biting for 1 week after application of the provisional restoration and treatment denture, but this ceased after occlusal adjustment. The treatment denture and provisional restoration were applied for 2 months and definitive prostheses prepared after confirming esthetics and the state of the periodontal tissue and occlusion. Full cast crowns were applied to \#14 and 15 and a labial facing crown made of composite resin to $\# 22$ as definitive prostheses. A removable partial denture with a metal base which would cover the attritioned area and act as a prosthetic for loss of \#11 and 21 was prepared. L oss of the incisors was restored using artificial teeth, and the morphology of the attritioned area of the maxillary dentition was restored by covering it with metal (Fig. 8). At the trial of the metal frame for the maxillary metal plate denture, the patient complained of discomfort due to the connector on the anterior palatal cover. The covered area was minimized by setting the posterior end of the connector between the 2 nd and 3 rd rugae of the transverse palatine fold. After confirming that the patient was satisfied with the reprepared metal frame, the artificial teeth were inserted and the definitive denture completed and applied (Fig. 9).

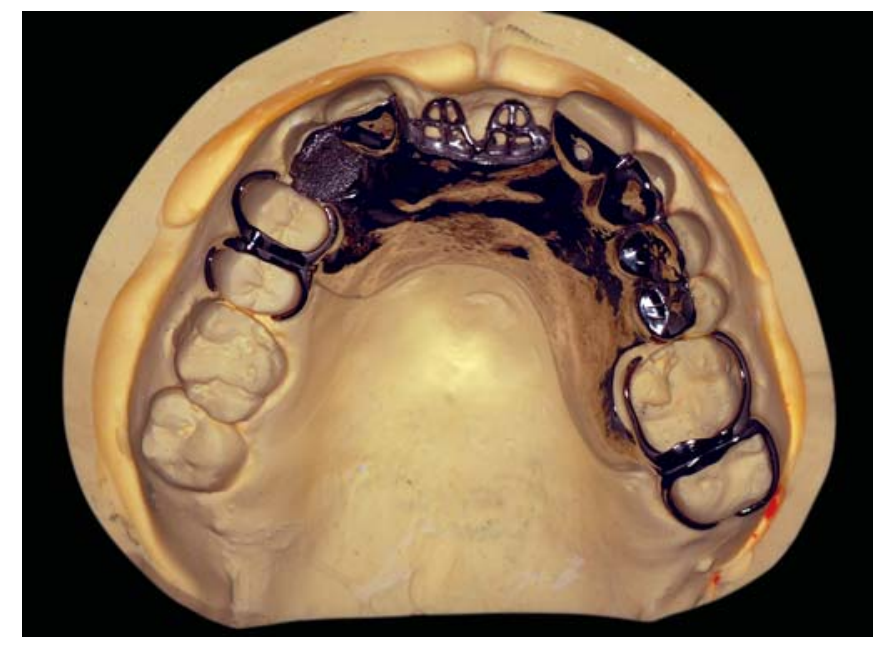

Fig. 8: Preparation of metal frame

For esthetic restoration in the maxillary incisor region, a labial facing crown made of composite resin to \#22 and a partial denture for loss of \#11 and \#21. Tooth \#13 was faced with composite resin and covered with the metal plate. The patient's chewing score was improved to $89 \%$, or level 5 , by appropriately adjusting the occlusal vertical dimension and oral rehabilitation, and masticatory dysfunction was alleviated. A nalysis of postoperative lateral cephal ograms revealed that the facial axis, facial depth, and mandibular arc had decreased by $0.5^{\circ}$ and that the lower facial height had increased by $1^{\circ}$ compared with preoperative values (Fig. 10). While no major problem arose concerning occlusal contact of the artificial teeth or compatibility of the impression surface for 1 year after prosthetic treatment, fracture of the resin face of \#13 was observed and repaired once. Presently, the patient is being followed up once every 

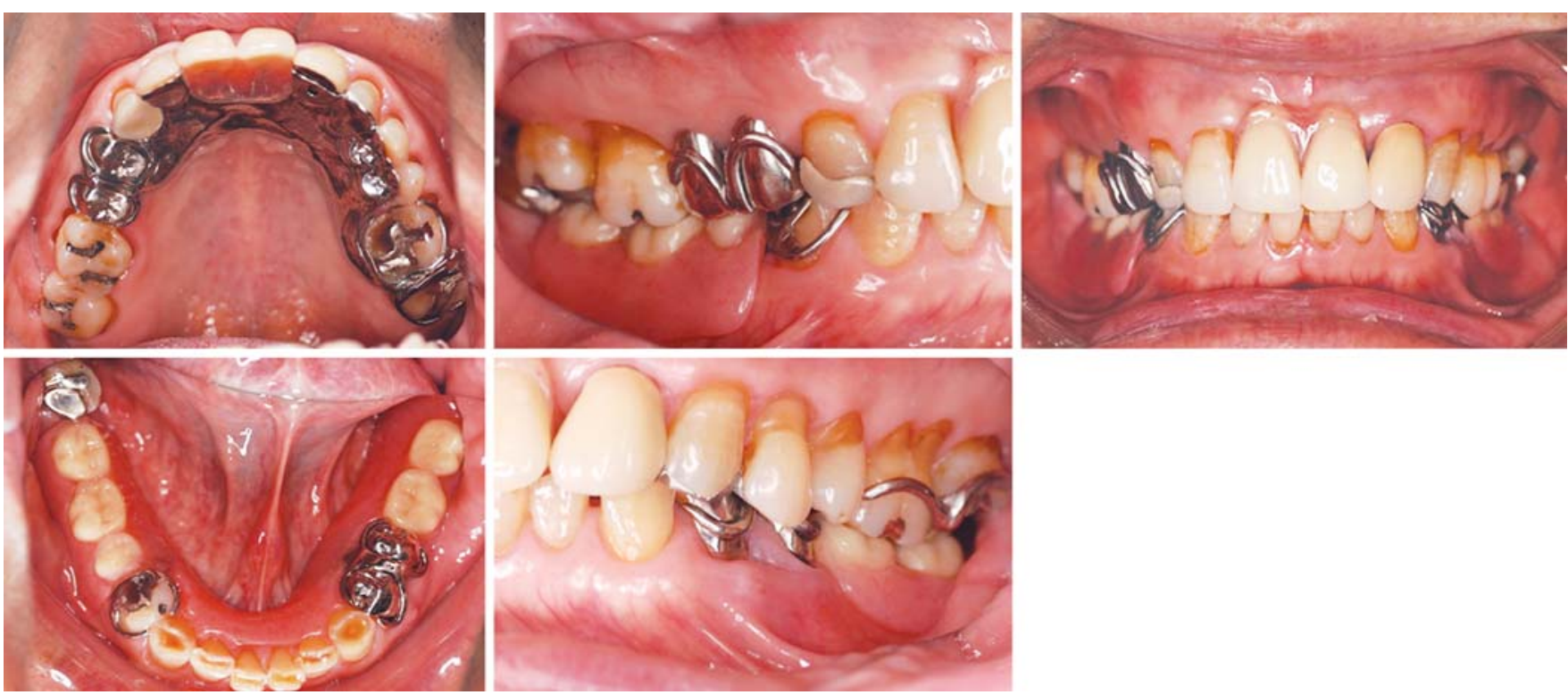

Fig. 9: Intraoral photographs of with definitive prosthesis applied

3 months. Plaque control and cleaning of the dentures are being practiced adequately. No caries has occurred in the remaining teeth, and no problem has arisen in the periodontal tissue.

\section{DISCUSSION}

In this patient, an increase in occlusal loading in the incisor region due to infraocclusion caused by dentures with an insufficient supporting ability and attrition of the remaining teeth induced detachment of the fixed partial denture in the maxillary incisor region and root fracture of tooth \#11. There are various methods of determining the appropriate vertical mandibular position in patients with a reduced occlusal vertical dimension. Here, the occlusal vertical dimension was increased by $5 \mathrm{~mm}$ between the subnasal point and submental region using the physiologic resting position of the mandible as a reference. Either a fixed or removable prosthesis must be selected in increasing vertical dimension in patients with infraocclusion due to attrition. If a fixed prosthesis is selected, there will be concerns over shortening of the abutment teeth. Although supplementary retention forms such as the box, groove, and parallel pin offer an effective means of retaining short abutment teeth, ${ }^{5}$ they were considered inappropriate in the present case due to severe attrition. Therefore, it is necessary to carry out crown lengthening accompanied by a surgical procedure or pulpectomy using a post and core if a fixed prosthesis is to be used in areas with severe attrition. ${ }^{6}$ However, such invasive treatment has become less popular recently with developments in techniques which require minimal or no preparation and new adhesives. ${ }^{7}$ Some studies have reported the use of a cap clasp or onlay type rest in treatment
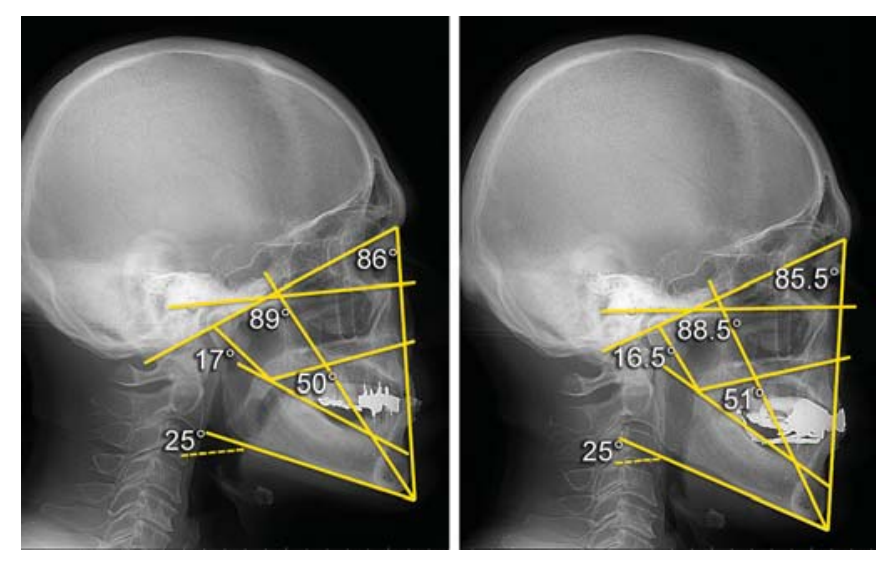

Fig. 10: Analysis of preoperative (left) and postoperative (right) lateral cephalograms

involving a removable prosthesis. ${ }^{8,9} \mathrm{~N}$ one of these approaches was considered appropriate in the present patient, however, due to the extent of attrition, number of maxillary teeth affected and loss of crown length. Therefore, a removable partial denture was selected to restore the morphology of the defect by covering the attritioned area. The patient reported frequent cheek biting for 1 week after vertical dimension was increased with a provisional restoration and treatment denture, probably due to the $5 \mathrm{~mm}$ increase in occlusal dimension, which may have destabilized occlusion. ${ }^{10}$ The patient stopped complaining of discomfort during chewing after application of the definitive prosthesis, however. Functional recovery was checked by comparing chewing function between before and after prosthetic treatment. M asticatory efficiency was improved by adjusting the occlusal vertical dimension and oral rehabilitation using a removable partial denture to cover the attritioned area. Decrease in the facial axis, facial depth, 
mandibular arc, and increase in the low er facial height were confirmed pre- and postoperatively to evaluate the occlusal vertical dimension. These changes are believed to indicate dilation of the mentum due to rotation of the mandibular condyle associated with increase in the occlusal vertical dimension. However, no objective preoperative evaluation of bruxism was made or the postoperative use of a night guard or night denture considered, even though the marked attrition observed here may have been caused by bruxism. ${ }^{11}$ Mildly invasive prosthetic treatment involved covering the attritioned surface with a metal denture without cutting the attritioned teeth. However, covering an attritioned surface provides an environment vulnerable to caries and periodontal disease, so careful plaque control is necessary. The surfaces of teeth showing attrition are at increased risk of hypersensitivity and caries. Therefore, an antihypersensitivity drug and fluoride were regularly applied. Moreover, facial fracture and abrasion of tooth \#13 and progression of attrition in the mandibular incisor region may induce change in anterior guidance, so careful follow-up is necessary.

\section{CONCLUSION}

$M$ asticatory dysfunction was restored and esthetic appearance improved by oral rehabilitation involving increase of vertical dimension and application of a removable partial denture to cover the attritioned area in a patient with infraocclusion due to attrition of the remaining teeth and loss of occlusal support in the molar region. Mildly invasive prosthetic treatment was performed by covering the attritioned surface with a metal base denture, not only infraocclusion but masticatory dysfunction and poor esthetics were al so treated.

\section{ACKNOWLEDGMENTS}

We would like to thank associate professor Jeremy Williams, Tokyo Dental College, for his assistance with the English of this manuscript.

\section{REFERENCES}

1. Carlsson GE, Johansson A, L undqvist S. Occlusal wear. A follow-up study of 18 subjects with extremely worn dentitions. A cta Odontol Scand 1985;43:83-90.

2. Ichikawa T, Sato H, K uboki T, Sato Y, Hideshima M, Y asuda $\mathrm{N}$, et al. Classification system for the completely dentate, partial and complete edentulism. J J ap A ss Dent Sci 2006;25:63-75.

3. Ricketts RM, Roth RH, Chaconas SJ, Schulhof RJ, Engle GA. Orthodontic diagnosis and planning. Denver: Rocky M ountain Data Systems 1982;107-12

4. Sato $Y, M$ inagi $S, A$ kagawa $Y, N$ agasawa $T$. A n evaluation of chewing function of complete denture wearers. J Prosthet D ent 1989;62:50-53

5. Johansson A, J ohansson AK, O mar R, Carlsson GE. Rehabilitation of the worn dentition. J Oral Rehabil 2008;35:548-66.

6. Setchell DJ. Conventional crown and bridgework. Br Dent J 1999;187:68-74.

7. Bartlett DW . Erosion and tooth surface loss. Int J Prosthodont 2003;16 (Suppl):87-88.

8. Takayama Y, Ichkawa M, Hosoi T. Prosthesis made of an onlaytype rest with high retentive force (cap clasp). Proshodont Res Pract 2007;6:132-37.

9. $\mathrm{Y}$ amamoto $\mathrm{H}$. C onstruction of a removable denture for a partially edentulous patient with infraocclusion. A nn Jpn Prosthodont Soc 2010;2:291-94.

10. Carlsson GE. Ingervall B, K ocak G. Effect of increasing vertical dimension on the masticatory system in subjects with natural teeth. J Prosthet Dent 1979;41:184-89.

11. Johansson A, Omar R, Carlsson GE. Bruxism and prosthetic treatment: A critical review. J Prosthodont Res 2011;55:127-36.

\section{ABOUT THE AUTHORS}

\section{Akinori Tasaka (Corresponding Author)}

Senior A ssistant Professor, D epartment of Removable Prosthodontics and Gerodontology, Tokyo Dental College, Chiba, Japan, e-mail: atasaka@tdc.ac.jp

\section{Takayuki Ueda}

Associate Professor, Department of Removable Prosthodontics and Gerodontology, Tokyo Dental College, Chiba, Japan

\section{Kaoru Sakurai}

Professor and Chair, Department of Removable Prosthodontics and Gerodontology, Tokyo Dental College, Chiba, Japan 